\title{
Spontaneous remission of obstructive jaundice in rats: Selection of experimental models
}

\author{
YUNFU LV ${ }^{1}$, JIE YUE ${ }^{1}$, XIAOGUANG GONG ${ }^{1}$, XIAOYU HAN ${ }^{1}$, HONGFEI WU ${ }^{1}$, JIE DENG ${ }^{1}$ and YEJUAN LI $^{2}$ \\ ${ }^{1}$ Department of General Surgery, Hainan Provincial People's Hospital, Haikou, Hainan 570311; ${ }^{2}$ Department of \\ Reproductive Medicine, Maternal and Child Health Care Hospital, Haikou, Hainan 571000, P.R. China
}

Received September 30, 2017; Accepted February 16, 2018

DOI: $10.3892 /$ etm.2018.6119

\begin{abstract}
The aim of the present study was to evaluate the prevalence and causes of spontaneous remission of obstructive jaundice in rats. Healthy male and female Wistar rats (180-220 g) were randomly assigned to receive common bile duct ligation (CBDL) and transection (group A), CBDL only (group $\mathrm{B}$ ), or CBD dissection without ligation or transection (control group $C ; n=36$ in each group). There was a difference in eye and skin jaundice prevalence between groups $\mathrm{A}$ and $\mathrm{B}$ from 14 days after surgery. The level of total bilirubin (TB) did not continue to increase in group $\mathrm{A}$ and began to decrease in the majority of rats in group $\mathrm{B}(\mathrm{P}<0.05$ vs. group $\mathrm{B})$. At day 21 after surgery, the TB level returned to normal in group B and no significant difference was observed compared with group C. At day 21 after surgery, significant dilatation of bile ducts above the ligature was observed in group A following cholangiography with $38 \%$ meglumine diatrizoate and this contrast agent did not spread to other sites. Slight dilatation of the proximal bile ducts was observed in group B and the contrast agent entered the intestinal lumen through the omental ducts adhering to the porta hepatis. After 14 days of surgery, there were 36 rats in group $\mathrm{A}$ and $\mathrm{B}$, and 17 rats exhibited spontaneous regression of jaundice. Overall, 47.2\% (17/36) of rats experienced spontaneous remission of obstructive jaundice, $82.4 \%$ (14/17) of which underwent ligation only. The spontaneous remission of jaundice may have been caused by shunting through very small bile ducts or omental ducts adhering to the porta hepatis. If a model of biliary obstruction is to be established in future research, a model of CBDL and transection is preferable. In this case, jaundice reduction surgery should be performed 14 days after establishment of the model.
\end{abstract}

Correspondence to: Dr Yunfu Lv, Department of General Surgery, Hainan Provincial People's Hospital, 19 Xiuhua Road, Xiuying, Haikou, Hainan 570311, P.R. China

E-mail: yunfu_lv@126.com

Key words: common bile duct, ligation, rats, spontaneous remission of jaundice, model selection

\section{Introduction}

Rats are used widely in experimental studies due to their small size, low cost, high vitality and short breeding cycle (1). In experimental research, rats are used to construct models of biliary obstruction and are subjected to external drainage of the orbiliary enterostomy (2-4). There are two primary ways to construct a biliary obstruction model: Opening the common bile duct and performing silk ligation and simple silk ligation alone $(5,6)$. The former requires tissue area separation, which is comprised of a large area and is time-consuming to perform (7). However, the surgery is thorough and the latter tissue separation area is relatively small (7). The surgical method, is simple owing to the fact that the common bile duct does not to be cut, only tied; however, loose ligating lines may cause the bile duct obstruction model to function less efficiently (8). At present, regardless of the surgical method used to establish the model of biliary obstruction, jaundice eliminating surgery is performed at the onset of jaundice (7), in order to establish the relevant indicative changes of jaundice prior to and following surgery $(9,10)$. Previous studies have not assessed whether obstructive jaundice subsides naturally (7). However, it has been observed that when creating a rat model of biliary obstruction, jaundice is corrected spontaneously, without the remission of biliary obstruction through surgery (11). In a considerable number of rats, if surgery for jaundice reduction is performed at this time, the results are likely to be compromised due to the condition naturally subsiding. Previous studies have demonstrated that natural jaundice may subside; however the causes of this spontaneous remission remain unknown (11-13). To the best of our knowledge, no previous studies exist that have elucidated this mechanism. Therefore, the aim of the current study was to investigate the prevalence and causes of spontaneous remission of obstructive jaundice. To the best of our knowledge, the present study revealed for the first time the incidence, timing and major types of jaundice that spontaneously dissipate in biliary obstruction modeled rats. Three causes of spontaneous regression of jaundice (microcirculation, adventitia of the adherent omental canal and loosening of the ligation) were analyzed. In particular, it was demonstrated by angiography that the small bile ducts of rats can be opened and that the small ducts present in the adhesions of the porta hepatic can communicate with the intestine. This discovery has not been 
previously described in animal experiments. This innovative discovery not only fills in the gaps in animal experiments, but also enriches the content of teaching materials. At the same time, it may exist as an important reference value for the selection of animal experiment models for clinical jaundice analysis and treatment application.

\section{Materials and methods}

Ethical approval. All researchers participating in this study were experts and graduate students from Hainan Provincial People's Hospital (Hainan, China). The study protocol was approved by the Hainan Provincial Science and Technology Commission of China (approval no. ZDYF2016158).

Groupings. A total of 108 healthy (54 male, 54 female), 8 month old, Wistar rats (180-220 g) were obtained from the Hainan Provincial Animal Laboratory and assigned randomly to receive common bile duct ligation (CBDL) and transection (group A), CBDL only (group B), or CBD dissected free without ligation or transection (control group $C ; n=36$ in each group). Rats were housed at a temperature of $20-25^{\circ} \mathrm{C}$ and a humidity of $50-65 \%$ with a $12 \mathrm{~h}$ light/dark cycle. Rats also received ad libitum access to rat-specific grain and water.

Model creation. Rats were fasted without water for $12 \mathrm{~h}$ prior to surgery. The rats were anesthetized (sodium pentobarbital, $40-45 \mathrm{mg} / \mathrm{kg}$, i.p.) and the fur at the incision area was removed or cut flat. All the fur on the abdomen was cleaned and disinfected with $75 \%$ alcohol prior to surgery. The upper abdomen was opened by a median incision. The CBD was located along the hepatoduodenal ligament and dissected free. For group A, the CBD was doubly ligated near the duodenum with 4-0 silk sutures and transected between the two ligatures. For group B, the CBD was doubly ligated near the duodenum without transection. For group C, the CBD was dissected free without ligation or transection.

Determination of indices. Blood was collected under anesthesia from the tail vein of six rats from each group at 1, 3, 7, 14, 21 and 28 days after surgery to determine the levels of total bilirubin (TB; Total Billirubin kit; cat. no. 0617071; Maccura Biotechnology Co., Ltd., Chengdu, China), direct bilirubin (DB; Direct Bilirubin Assay kit; Maccura Biotechnology Co., Ltd.; cat. no. 0817081), total bile acid (TBA assay kit; Maccura Biotechnology Co., Ltd.; cat. no. 0611031) and alanine aminotransferase (ALT assay kit; Maccura Biotechnology Co., Ltd. Cat. no. 0617031). Following opening of the abdomen (conducted after surgery), the CBD diameter was measured and a small piece of liver tissue was collected for histopathology.

At day 21 after surgery, rats with spontaneous remission of jaundice underwent cholangiography by injection of $38 \%$ meglumine diatrizoate (MD) into the left or right hepatic duct using a size-7 needle and MD distribution was observed via $\mathrm{X}$-rays 21 days following surgery to ascertain the cause of jaundice remission.

At day 28 after surgery, $0.2 \mathrm{ml}$ of technetium-99m iminodiacetic acid ( ${ }^{99} \mathrm{~m}$ TC-IDA) was injected into the tail vein and its distribution in the bile ducts was observed using an isotope scanner (ecom; Siemens AG, Munich, Germany).
Rats were anesthetized using $40 \mathrm{mg} / \mathrm{kg}$ intraperitoneal $1 \%$ sodium pentobarbital and then sacrificed via exsanguination. Tissues of equal size were removed from bile ducts above and below the ligature. The distribution of rat $99 \mathrm{mTC}$-IDA was assessed whilst rats were exposed to low-dose anesthesia using a single-photon emission computerized tomography isotope scanner (Siemens AG) with radiocolloid method software included. The omentum was adhered to the porta hepatis, jejunum and ileum to determine ${ }^{99 m}$ TC-IDA levels pot-sacrifice.

Statistical analysis. Data were analyzed using SPSS 18.0 (SPSS, Inc., Chicago, IL, USA). Levels of TB, DB, TBA and ALT were compared among and within groups using one-way analysis of variance, followed by a post hoc LSD test, and presented as the mean \pm standard deviation. Radionuclide quantitation was compared using a paired Student's t-test. $\mathrm{P}<0.05$ was considered to indicate a statistically significant difference.

\section{Results}

General observations. At day 3 after surgery, rats in groups A and B were listless and had poor appetite, with slightly yellow eyes and tails. Slightly yellow abdominal organs, omental adhesions to the porta hepatis and slight dilatation of proximal bile ducts were also observed.

At day 7 after surgery, obviously yellow ears, eyes, tails and internal organs were observed (Fig. 1), as well as dense hilar adhesions, dilatation of proximal bile ducts and a moderate liver hardness in the majority of the rats in groups A and B. In each group, there was 1 rat in which jaundice was not observed.

At day 14 after surgery, a more intense yellow color of the ears, eyes and tail was noted, as were ascites, a hard liver, orange abdominal organs and marked dilatation of the proximal CBD (maximum diameter, $20 \mathrm{~mm}$ ) in 15 of the 18 rats (Fig. 2; from 14-28 days following surgery; $n=6$ per group) in group A. Slightly less yellow ears, eyes and tail, along with an improved activity and appetite, less yellow abdominal organs, no significant ascites, a softened liver, undetached bile duct ligatures, non-significant dilatation of proximal bile ducts, and severe omental adhesions to the porta hepatis were observed in 3 rats (16.7\%) in group A and 14 rats (77.8\%) in group B. Increased jaundice was observed in an additional 4 rats in group $\mathrm{B}$, with abdominal findings similar to the aforementioned 15 rats in group A.

At day 21 after surgery, persistent jaundice and poor mental status were observed in 15 rats in group A; however, the other 3 rats in group A and 14 rats in group B exhibited complete remission of jaundice of the ears, eyes, tail and abdominal organs, and a soft liver texture (Fig. 3), as well as no dilatation of proximal bile ducts, weight gain and a normal appetite.

At day 28 after surgery, 2 rats with jaundice in group A died; persistent jaundice, poor mental status and low levels of activity were observed in the 4 remaining rats in group $\mathrm{A}$ and 4 rats with jaundice in group B. The remaining 14 rats in group B and 3 rats in group A exhibited no jaundice and had normal hair color, good mental status, normal appetite and weight gain. Group C indicators, including skin, coat, color, mental status and degree of activity were normal. Incision infection or abdominal infection did not occur in any rats. 


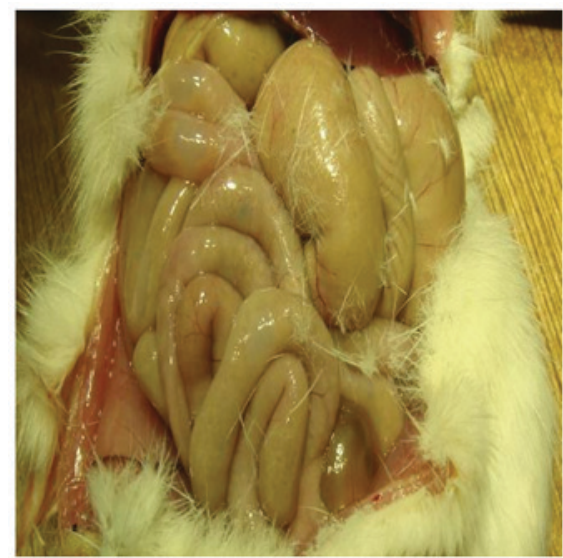

Figure 1. Representative image of a group A mouse with notable yellowing of the abdominal organs.

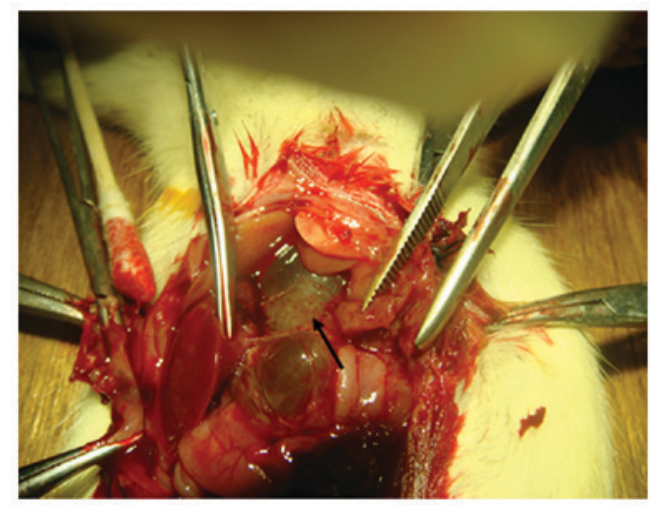

Figure 2. Representative image of a group A mouse with notably dilated common bile duct (black arrow indicates expansion of the proximal common bile duct).

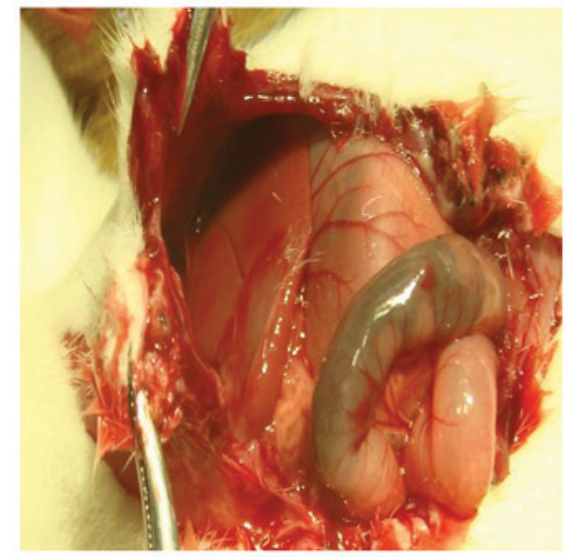

Figure 3. Representative image presenting reduced yellowing of abdominal organs in group B.

Serum level of bilirubin. There were significant differences in TB level among the three groups $(\mathrm{P}<0.05)$. The TB level was significantly different among days 1,3 and 7 in groups $\mathrm{A}$ and $\mathrm{B}$ $(\mathrm{P}<0.05)$. A significant increase in $\mathrm{TB}$ level was noted in group $\mathrm{A}$ compared with group $\mathrm{C}$ at all time points $(\mathrm{P}<0.05)$. A significant increase in $\mathrm{TB}$ was observed in group $\mathrm{B}$ compared with group $\mathrm{C}$ at $1,3,7$ and 14 days $(\mathrm{P}<0.05)$, but not at 21 or 28 days (Fig. $4 \mathrm{~A})$.
No significant difference was identified in the DB level at 14, 21 and 28 days in group A, but a significant difference was observed at the same time points in group $\mathrm{B}(\mathrm{P}<0.05)$. There was a significant increase in DB level in group A compared with group $\mathrm{C}$ at all time points $(\mathrm{P}<0.05)$. There was a significant increased in DB level in group B compared with group $\mathrm{C}$ at $1,3,7$ and 14 days $(\mathrm{P}<0.05)$, but not at 21 or 28 days (Fig. $4 \mathrm{~B})$. The TBA level at 1, 3 and 7 days were significantly different from that at 14, 21 and 28 days comparing groups $\mathrm{A}$ and $\mathrm{C}$, and $\mathrm{B}$ and $\mathrm{C}(\mathrm{P}<0.05)$; however no significant difference was observed in group B when compared with group $\mathrm{C}$ at 21 and 28 days (Fig. 4C).

ALT level. ALT levels increased, 1 day following surgery. Then, the ALT level decreased, with the sharpest decrease from days 2 to 3 and a slightly smaller decrease from days 4 to 7. The ALT level decreased to a normal level after day 14. When comparing groups $\mathrm{A}$ and $\mathrm{C}$, and B and C, the ALT level significantly decreased between days 1 and $3(\mathrm{P}<0.05)$. There was no significant difference between days 7 and 14 ( $\mathrm{P}>0.05)$. The radionuclide level from the bile ducts above the ligature was significantly different from those at sites with the omentum was adhered to the porta hepatis and ileum in group A and B when compared with group $\mathrm{C}(\mathrm{P}<0.05)$. The ALT level was significantly higher in groups $\mathrm{A}$ and $\mathrm{B}$ compared with group $\mathrm{C}$ at days 1 and $3(\mathrm{P}<0.05)$, but no significant differences were observed from day 7 onwards (Fig. 4D).

Cholangiography. Groups A and B underwent cholangiography with MD treatment at day 21 after surgery. Marked dilatation of the bile ducts above the ligature was observed in group A, with a maximum diameter of $20 \mathrm{~mm}$ and the contrast agent did not spread to other sites (Fig. 5). Slight dilatation of the bile ducts was observed in group B and the contrast agent entered the small intestinal lumen through the omentum adhering to the porta hepatis (Fig. 6).

Radionuclide imaging and quantification. At day 28 after surgery, ${ }^{99 \mathrm{~m} T C}$-IDA was injected into the tail vein of 6 rats with significant jaundice in group A and 6 rats with remission of jaundice in group B. The radionuclide accumulated first in the liver. In group A, it did not enter the intestine and images of the bladder were obtained in a small number of rats (Fig. 7). However, in group B, ${ }^{99 \mathrm{~m}}$ TC-IDA started to enter the intestine from the liver through the omentum adhering to the porta hepatis 5 min after administration to produce radionuclide images. Images of the bladder were obtained in a small number of rats (Fig. 8).

The radionuclide level at site from the bile ducts above the ligature was significantly different from those at sites where the omentum was adhered to the porta hepatis, jejunum, and ileum in group A (all $\mathrm{P}<0.05)$ but not in group $\mathrm{B}$. The radionuclide level at site bile ducts above the ligature in group A was significantly higher compared with group $\mathrm{B}(\mathrm{P}<0.01)$. At these sites, the isotopic abundance of group B was significantly higher than that of group A as 99mTC-IDA had entered group $\mathrm{B}$ tissue. $(\mathrm{P}<0.05$; Fig. 9).

Histopathology of the liver. On day 1 after surgery, cholestasis, hepatocyte swelling, translucent cytoplasm, steatosis and 
A

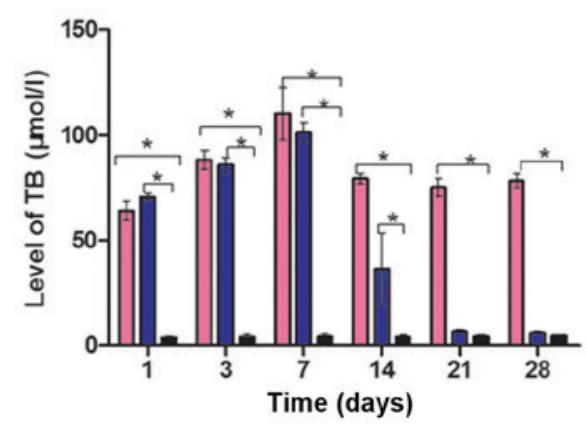

C

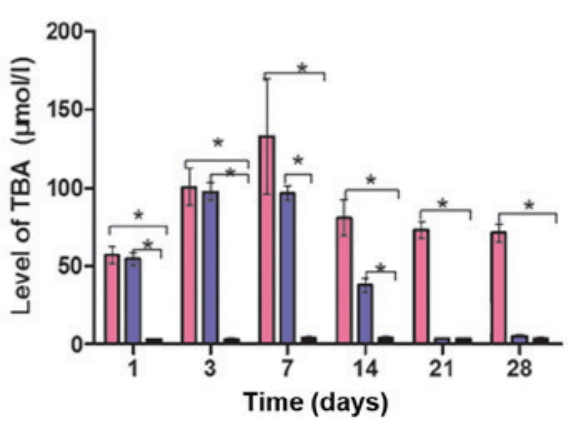

B

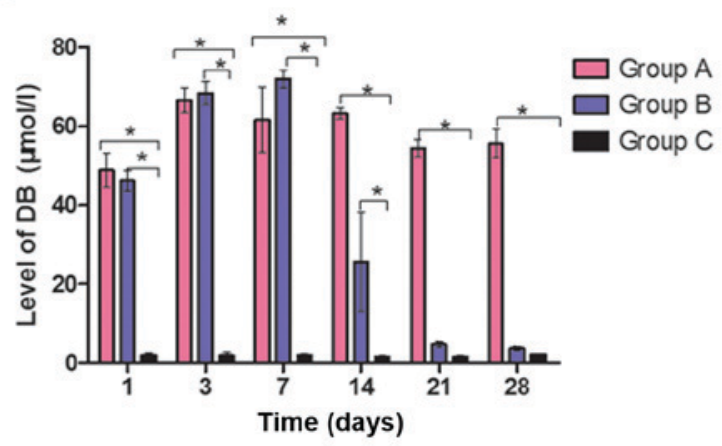

D

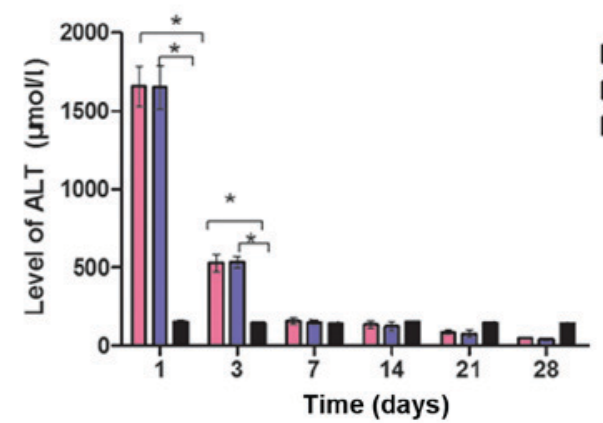

Figure 4. Changes in liver function from days 1 to 28 post-surgery. Changes of (A) TB, (B) DB, (C) TBA and (D) ALT. "P<0.05. TB, total bilirubin; DB, direct bilirubin; TBA, total bile acid; ALT, alanine aminotransferase.

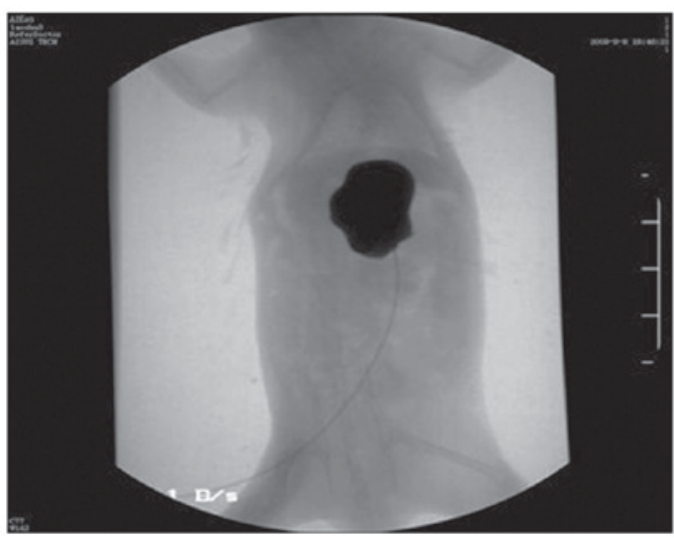

Figure 5. No diffusion of contrast agent in bile ducts in an X-ray image of a group A rat.

eosinophilic changes, as well as punctate and focal necrosis were observed in groups A and B.

In groups A and B, 7-14 days after surgery, dilatation and hyperplasia of the small bile ducts in the portal area, marked hepatic necrosis, marked collagen deposition, compression of hepatic sinusoids, and dilatation and congestion of interlobular vessels were observed (Fig. 10).

At $\geq 21$ days after surgery, continued hyperplasia of bile ducts and liver fibrosis were observed in group A. Reduced hyperplasia of bile ducts, relatively reduced collagen deposition, non-marked dilatation and congestion of interlobular vessels were observed in group B (Fig. 11), which suggested markedly reduced liver fibrosis. These findings were consistent with the changes observed in serum indices.

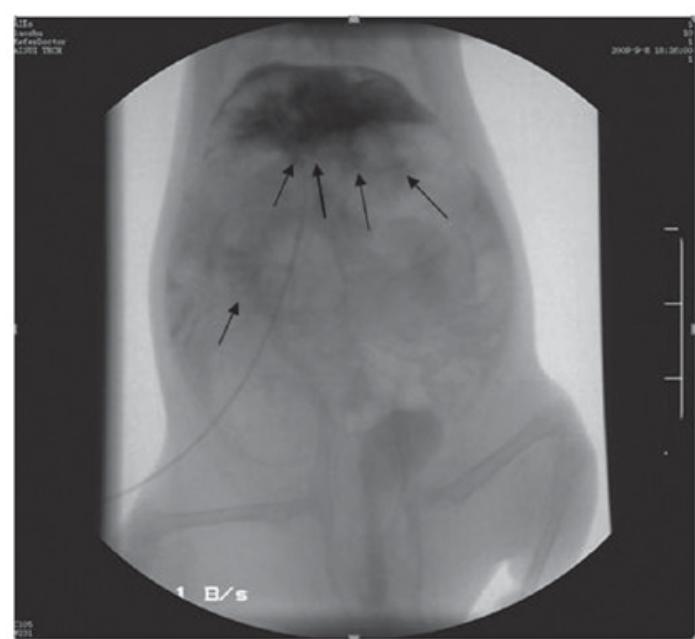

Figure 6. Passage of contrast agent from intrahepatic bile ducts into the intestinal lumen via three ducts adhering to the omentum in the porta hepatis in X-ray images of group B. Black arrow indicates contrast agents that flow into the gut, demonstrating that bile may enter the gut lumen via these tubes.

\section{Discussion}

Serum levels of TB and DB increased progressively from 1 day after common bile duct ligation and peaked at 7 days. The serum level of bilirubin continued to increase in most rats in group A 7 days after surgery, whereas it began to decrease 14 days after surgery and decreased substantially to normal levels 21 days after surgery in most rats in group B. This is similar to the time-to-reduction of the serum level of bilirubin reported by Huang et al (14). McIntyre and Rosalki (15) 


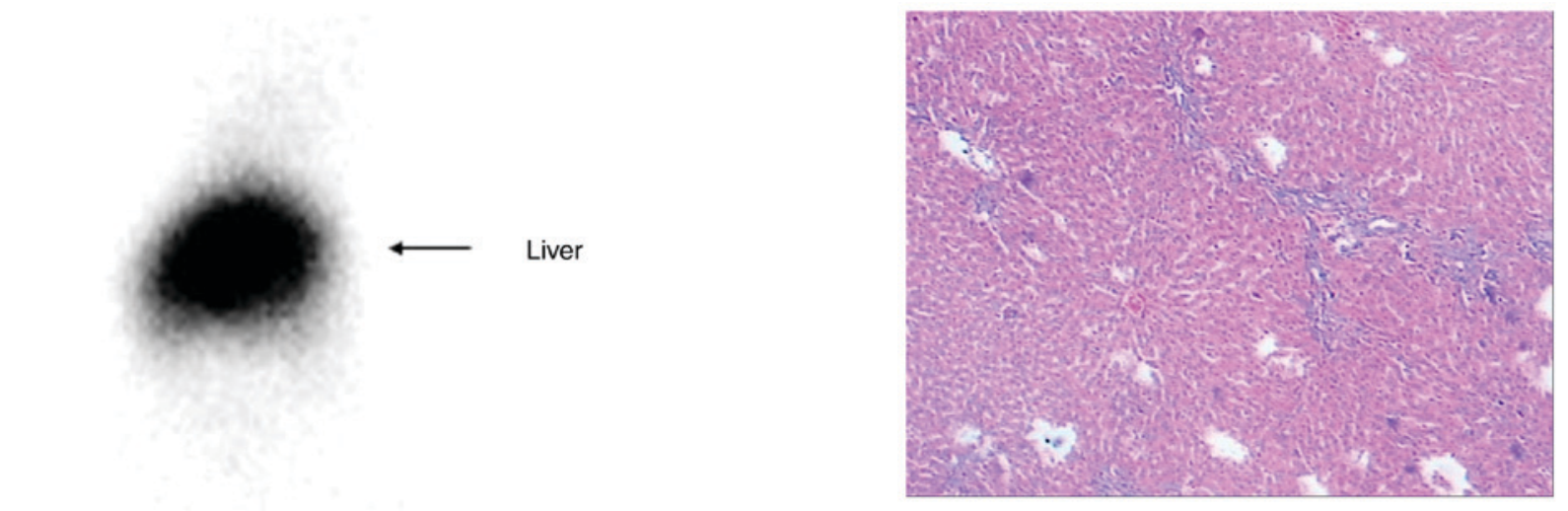

Figure 10. Histopathology staining at day 14 after surgery from a group A rat (magnification, x200). Features include: Dilatation and hyperplasia of the small bile ducts in the portal area, marked hepatic necrosis, marked collagen deposition, compression of hepatic sinusoids, and dilatation and congestion of interlobular vessels.

Figure 7. Radionuclide image in group A.

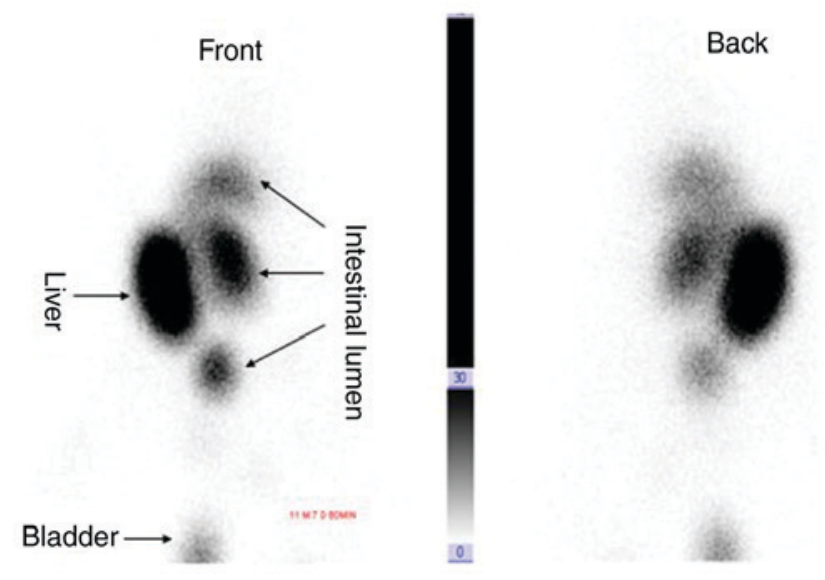

Figure 8. Radionuclide images in group B.

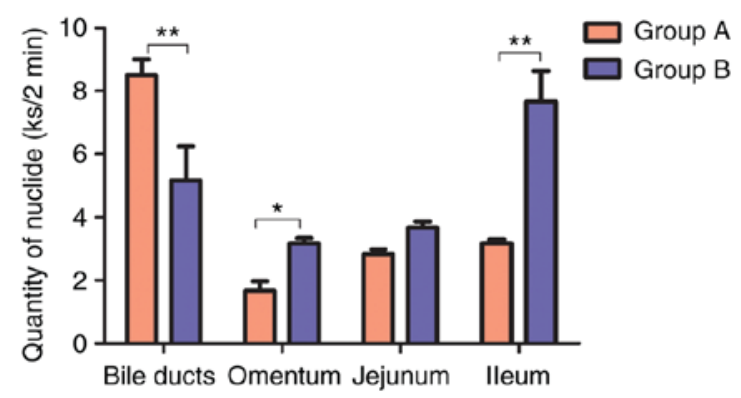

Figure 9. Quantity of radionuclide in different anatomic areas. " $\mathrm{P}<0.05$, ${ }^{* *} \mathrm{P}<0.01$. Ks, count per sec.

reported that the serum level of bilirubin generally increases up to $170-500 \mu \mathrm{mol} / 1$ after complete biliary obstruction, and does not continue to increase.

The change in TBA level was different from that of bilirubin. It Significantly increased 1 day after surgery in groups A and $\mathrm{B}$. The TBA level did not decrease and remained high in group A 14 days after surgery. Increased levels of DB and TBA reflected bile obstruction $(16,17)$. The TBA level decreased in

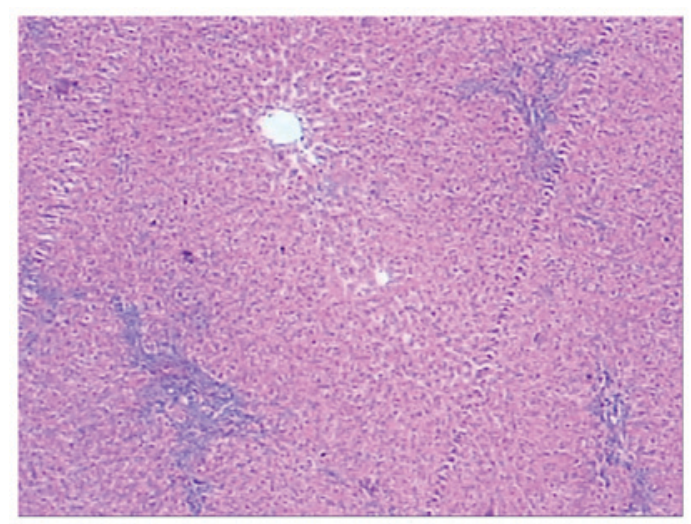

Figure 11. Histopathology staining from group B at day 21 after surgery (magnification, x200). There is reduced hyperplasia of bile ducts, relatively reduced collagen deposition, non-marked dilation and congestion of interlobular vessels.

group B 14 days after surgery and decreased to a normal level at 21-28 days, but did not fall below the normal level.

The causes of spontaneous remission of obstructive jaundice in rats are not known. Wu et al (11) proposed that it may be caused by increased pressure within the bile duct, which leads to compression of the portal vein and hepatic artery. This phenomenon leads to reduced blood flow in the liver, severe liver dysfunction, impaired ability of hepatocytes to secrete bile, and reduced regurgitation of bile into blood.

Numerous animal and clinical studies have investigated the mechanism of liver injury and pathophysiological changes during obstruction of the bile duct $(18,19)$. These studies have confirmed pathological liver injury in rats with obstructive jaundice. The changes in enzyme levels and liver histology observed in the present study also confirmed pathological liver injury following biliary obstruction, but do not explain the causes of remission of spontaneous jaundice.

There are three possible causes of remission of spontaneous jaundice. The first possible cause is compensatory opening of very small bile ducts (VSBDs). The hepatoduodenal ligament of the rat contains the CBD and potentially numerous VSBDs (20). Following CBDL, the VSBDs may 
open to compensate, thereby restoring bile flow. This phenomenon may explain the complete absence of jaundice following CBDL in 2 rats in the present study. The basic premise of the Kasai procedure (21) to treat biliary atresia is that there may be VSBDs in the vicinity of the porta hepatis even with extrahepatic biliary atresia, and appropriate resection of the fibers in the porta hepatis that compresses the VSBDs may allow bile discharge and patient survival. Kordzaia and Jangavadze (22) injected ink into dilated proximal bile ducts, and identified that previously unknown VSBDs were distributed along the veins in the adventitia.

The second possible cause of remission of spontaneous jaundice is passage into the intestine via omental ducts adhering to the liver edge. MD injected into dilated proximal bile ducts was observed to enter the small intestine via the omentum adhering to the liver edge. The image was unlike that of a hidden CBD with a clear linear contour, and instead exhibited several vessels that seemed to adhere to the omentum, via which the contrast agent had passed into the intestine. Furthermore, in rats with remission of jaundice, the radionuclide was discharged from the liver into the intestine via extensive omental ducts in the porta hepatis to gradually produce radionuclide images 5 min after ${ }^{99}$ TC-IDA injection via the tail vein. Comparison of radionuclide levels among different sites revealed no significant difference among bile in bile ducts above the CBD ligatures, in blood vessels adhering to the omentum in the porta hepatis, or in the intestines of rats with remission of jaundice, whereas the opposite was true for rats with persistent jaundice. These findings suggested that bile above the ligatures may pass into the intestine through omental ducts adhering to the porta hepatis in rats with remission of jaundice, although this has not been supported by pathological evidence. The spontaneous remission of jaundice began 14 days after establishment of the model, when omental adhesions to the porta hepatis were likely to have been relatively stable, which would have been conducive to the shunting of bile. The mechanism of action sustaining the passage of bile into the intestine via ducts adhering to the omentum is not known and requires further research.

The third possible cause of remission of spontaneous jaundice is loose ligatures. Spontaneous remission of jaundice occurred predominantly in rats that received ligation only. Therefore, loose ligatures, for example due to untightened knots or short silk suture ends, may have been the cause. However, the ligatures were intact in the two groups. If the ligatures were loose in rats that received ligation only, cholangiography with MD should have exhibited images of the CBD with clear, broad lines. Loose ligatures in rats that underwent ligation and transection were associated with biliary peritonitis (23). The rats, in general, would not survive biliary peritonitis, and bile would be seen within the abdominal cavity of survivors. However, no bile was observed in the abdominal cavity of the rats that underwent ligation and transection in the present study. Loose ligatures theoretically exist, but should be evaluated specifically.

Overall, 47.2\% (17/36) of rats experienced spontaneous remission of jaundice (A group), 82.4\% (14/17) of which underwent ligation only (B group). The separation area may have been relatively small in rats that received ligation only, so VSBDs or hidden CBDs could not be transected or ligated readily, which was conducive to compensatory opening. Furthermore, the numerous blood vessels in the hepatoduodenal ligament could lead to adhesions. Therefore, $77.8 \%$ of rats experienced spontaneous remission of jaundice 14 days after surgery without external drainage or biliary enterostomy. In this case, the use of internal or external drainage to reduce jaundice will most likely provide inaccurate results. A total of $16.7 \%$ of rats that underwent ligation and transection also experienced spontaneous remission of jaundice, but the majority of rats did not experience spontaneous remission. This observation may have been because such rats had large separation areas, and VSBDs or hidden CBDs were likely to be transected and could not adhere to the greater omentum with ease. Therefore, CBD transection is the preferred model of biliary obstruction, and surgery may be undertaken to reduce jaundice 14 days after establishment of the model. If a ligation model is selected, to reflect the actual effects following jaundice reduction and restoration of normal morphology and physiological functions of the liver (24), jaundice-reduction surgery should be postponed by 1 week until 21 days after establishment of the model and performed in rats with persistent jaundice. This approach should be adopted since there is a 1-week period of jaundice reduction 14 days after establishment of the model, in addition to the high prevalence of spontaneous remission of jaundice. In conclusion, the establishment of a biliary obstruction model should be considered as the first choice for choledocholithotomy + ligation procedures. Yellow reduction surgery is a procedure in which jaundice is eliminated through internal and external drainage. The present study demonstrated that jaundice may spontaneously dissipate following 14 days, thus eliminating the necessity for surgery.

\section{Acknowledgements}

The authors would like to thank the Animal Experiment Center of Hainan Medical College (Hainan, China) for providing the experimental location and assisting in completing the experiments. The authors would also like to thank Professor Chenghui Luo (Teaching and Research Office of Statistics, Hainan Provincial Health School, Hainan, China) for completing statistical analyses for this study.

\section{Funding}

The study was supported by the Hainan Provincial Key Scientific and Technological Research and Development Projects (grant no. ZDYF2016158), and the Hainan Provincial Government for providing financial assistance (grant no. 288).

\section{Availability of data and materials}

All data generated or analyzed during this study is included in this published article and can be used as a reference by other scholars.

\section{Authors' contributions}

YLv designed the study, organized and implemented the experiment, observed the experimental results, collected and analyzed relevant information, and prepared and submitted 
the manuscript. JD and YLi conducted statistical analyses and prepared the figures. JY, XG XH and HW performed the experiments.

\section{Ethics approval and consent to participate}

The study protocol was approved by the Hainan Provincial Science and Technology Commission, China (approval no. ZDYF2016158).

\section{Consent for publication}

Not applicable.

\section{Competing interests}

The authors declare no competing financial interests.

\section{References}

1. Bulte JW, Schmieder AH, Keupp J, Caruthers SD, Wickline SA and Lanza GM: MR cholangiography demonstrates unsuspected rapid biliary clearance of nanoparticles in rodents: Implications for clinical translation. Nanomedicine 10: 1385-1388, 2014.

2. Ferreira MA, Santos JS, Dutra RA, Salgado W Jr, Kemp R, Domiciano C, Ramalho LN, Sankarankutty AK and Castro-eSilva Od: Bilioduodenal anastomosis in rats with extra-hepatic biliary obstruction is followed by lesions ischemia and reperfusion-induced. Acta Cir Bras 23 (Suppl 1): S47-S52, 2008.

3. Kemp R, de Araújo WM, de Castro AA, Ardengh JC, Neder L and dos Santos JS: Influence of biliary drainage on the repair of hepatic lesions in biliary fibrosis. J Surg Res 169: e127-e136, 2011.

4. Wang ZK, Xiao JG, Huang XF, Gong YC and Li W: Effect of biliary drainage on inducible nitric oxide synthase, CD14 and TGR5 expression in obstructive jaundice rats. World $\mathrm{J}$ Gastroenterol 19: 2319-2330, 2013.

5. Li JY, Zhu X and Fu ZM: Experimental study of postoperative obstructive jaundice and postoperative care. Jiangxi Yixueyuan Xuebao 31: 73-76, 1991 (In Chinese).

6. Saiki S, Chijiiwa K, Komura M, Yamaguchi K, Kuroki S and Tanaka M: Preoperative internal biliary drainage is inferior to external biliary drainage in liver regeneration and function after hepatecctomy in obstruction jaundice rats. Ann Surg 230: 655-662, 1999

7. Su X, Luo KF, Wu L, Wu LL and Li Wen: The correlation study of the plasma diamine oxidase activities and the intestinal barrier function in rats with obstructive jaundice after internal and external biliary drainages. Xian Dai Sheng Wu Yi Xue Jin Zhan 17: 3216-3219, 2014 (In Chinese).

8. Gong $\mathrm{YH}$ and $\mathrm{Li} \mathrm{W}$ : The different effects of internal and external biliary drainages on the blood levels of endotoxin interleukin-2 and interleukin-6 in rats with obstructive jaundice. Weichangbingxue He Ganbingxue Zazhi 4: 329-331, 2008 (In Chinese)
9. Zeng Y, Zhang Y, Zheng S and Dong J: Establishment of model of selective external biliary drainage in rats with obstructive jaundice. Zhonghua Xiao Hua Wai Ke Za Zhi 6: 209-212, 2007 (In Chinese).

10. Zhang SH, Liao CX, Zhang CX, Deng H, Zhu H, Lei L and Yao C: Establishment of a mouse model of biliary obstruction and its dynamic observations. Nan Fang Yi Ke Da Xue Xue Bao 28: 1579-1581, 2008 (In Chinese).

11. Wu W, Yang Y and Huang Q: Bilirubin metabolism after obstructive jaundice in rats. Zhonghua Gan Dan Wai Ke Za Zhi 5, 288-291, 2001 (In Chinese).

12. Ni Y, Marchal G, Lukito G, Yu J, Mühler A and Baert AL: MR imaging evaluation of liver enhancement by Gd-EOB-DTPA in selective and total bile duct obstruction in rats: Correlation with serologic, microcholangiographic, and histologic findings. Radiology 190: 753-758, 1994.

13. Kountouras J, Scheuer PJ and Billing BH: Effect of prolonged bile duct obstruction in the rat on hepatic transport of bilirubin. Clin Sci (Lond) 68: 341-347, 1985.

14. Huang Q, Liu C, Zhu C, Xie F and Hu S: Postoperative anastomotic bile duct stricture is affected by the experience of surgeons and the choice of surgical procedures but not the timing of repair after obstructive bile duct injury. Int J Clin Exp Pathol 7: 6635-6643, 2014

15. McIntyre $\mathrm{N}$ and Rosalki S: Biochemical investigations in the management of liver disease. In: Hepatobiliary Diseases. Springer, Berlin, pp39-71, 1992.

16. Nehéz L and Andersson R: Compromise of immune function in obstructive jaundice. Eur J Surg 168: 315-328, 2002.

17. Scopa CD, Koureleas S, Tsamandas AC, Spiliopoulou I, Alexandrides T, Filos KS and Vagianos CE: Beneficial effects of growth hormone and insulin-like growth factor I on intestinal bacterial translocation, endotoxemia, and apoptosis in experimentally jaundiced rats. J Am Coll Surg 190: 423-431, 2000.

18. Sinenchenko GI, Kabanov Mlu and Tikhonchuk SV: Perspectives of using ozonized solutions in complex treatment of patients with mechanical jaundice. Vestn Khir Im I I Grek 163: 91-94, 2003 (In Russian).

19. Shibayama Y: Endotoxaemia and hepatic injury in obstructive jaundice. J Pathol 159: 335-339, 1989.

20. Huang Z and Zou S (eds): Extrahepatic biliary duct. In: Biliary Surgery. Beijing: People's Medical Publishing House, pp23-26, 2010 (In Chinese).

21. Lv Y, Han X, Gong X, Wu H, Liu N, Hu Y, Deng J, Li Y and Li Q: Etiological classification of bile duct dilatation and proportion of each cause in 1430 patients. Int J New Technol Res 1: 51-54, 2015.

22. Kordzaia D and Jangavadze M: Unknown bile ductuli accompanying hepatic vein tributaries (experimental study). Georgian Med News 234: 121-129, 2014.

23. Lv Y and Dang J: Diagnostics and therapeutics of intra-extrahepatic cholangiectasis (Chinese edition). Science Press 1: 319-321, 2014.

24. Tag CG, Weiskirchen S, Hittatiya K, Tacke F, Tolba RH and Weiskirchen R: Induction of experimental obstructive cholestasis in mice. Lab Anim 49 (1 Suppl): 70-80, 2015.

(i) (5) This work is licensed under a Creative Commons Attribution-NonCommercial-NoDerivatives 4.0 International (CC BY-NC-ND 4.0) License. 